Journal of

Applied

Crystallography

ISSN 1600-5767

\title{
High-resolution synchrotron diffraction study of porous buffer InP(001) layers
}

\author{
Andrey A. Lomov, Vasily I. Punegov, Dusan Nohavica, Mikhail A. Chuev, \\ Alexander L. Vasiliev and Dmitrii V. Novikov
}

J. Appl. Cryst. (2014). 47, 1614-1625

Copyright (C) International Union of Crystallography

Author(s) of this paper may load this reprint on their own web site or institutional repository provided that this cover page is retained. Republication of this article or its storage in electronic databases other than as specified above is not permitted without prior permission in writing from the IUCr.

For further information see http://journals.iucr.org/services/authorrights.html

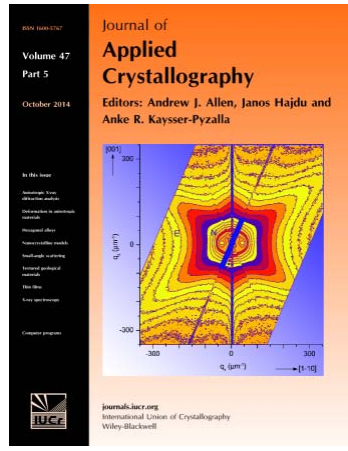

Many research topics in condensed matter research, materials science and the life sciences make use of crystallographic methods to study crystalline and non-crystalline matter with neutrons, X-rays and electrons. Articles published in the Journal of Applied Crystallography focus on these methods and their use in identifying structural and diffusioncontrolled phase transformations, structure-property relationships, structural changes of defects, interfaces and surfaces, etc. Developments of instrumentation and crystallographic apparatus, theory and interpretation, numerical analysis and other related subjects are also covered. The journal is the primary place where crystallographic computer program information is published.

Crystallography Journals Online is available from journals.iucr.org 


\section{Journal of \\ Applied \\ Crystallography \\ ISSN 1600-5767}

Received 23 May 2014

Accepted 15 July 2014

(C) 2014 International Union of Crystallography

\section{High-resolution synchrotron diffraction study of porous buffer InP(001) layers}

\author{
Andrey A. Lomov, ${ }^{\text {a* Vasily I. Punegov, }}$, Dusan Nohavica, ${ }^{\text {c }}$ Mikhail A. Chuev, \\ Alexander L. Vasiliev ${ }^{d}$ and Dmitrii V. Novikov
}

\begin{abstract}
a Laboratory of Surface Physics and Microelectronic Structures, Institute of Physics and Technology of Russian Academy of Science, Nahimovskii prospekt 36/1, Moscow 117218, Russian Federation, b Laboratory of Theoretical and Computing Physics, Department of Mathematics, Komi Science Center of Ural Division of RAS, Kommunisticheskaya 24, Syktyvkar 167001, Russian Federation, ${ }^{\mathrm{c}}$ Department of Materials and Diagnostics, Institute of Photonics and Electronics of ASCR, Chaberska 57, Prague 18251, Czech Republic, 'Laboratory of Electron Microscopy, Shubnikov Institute of Crystallography of RAS, Leninskii prospekt 59, Moscow 119333, Russian Federation, and ${ }^{\mathbf{e}}$ Photon Science, Deutsches Elektronen-Synchrotron DESY, Notkestrasse 85, Hamburg D-22603, Germany. Correspondence e-mail: lomov@ftian.ru
\end{abstract}

X-ray reciprocal space mapping was used for quantitative investigation of porous layers in indium phosphide. A new theoretical model in the frame of the statistical dynamical theory for cylindrical pores was developed and applied for numerical data evaluation. The analysis of reciprocal space maps provided comprehensive information on a wide range of the porous layer parameters, for example, layer thickness and porosity, orientation, and correlation length of segmented pore structures. The results are in a good agreement with scanning electron microscopy data.

\section{Introduction}

Future advancement in micro- and optoelectronics is directly dependent on progress in the technology of defect-free semiconductor heterostructures, which demonstrate very high values of charge concentration and mobility in the active layers (Landolt-Börnstein, 1982; Adachi, 1992). The range of attainable properties and compositional design of heterostructures is strongly limited by the mismatch of lattice parameters in the used materials. The mechanical strains caused by this mismatch trigger the formation of dislocations on the layer boundaries and threading dislocations inside the layers, which leads to degradation of electrophysical and optical properties. To avoid this problem, buffer layers are often used. The buffer layers can be of different types, including gradient (Katcki et al., 1999; Choi et al., 2009), metamorphous (Zeng et al., 2001; Hoke et al., 2003) or porous (Soldatenkov et al., 1999; Arsentyev et al., 2002, 2005; Sitnikova et al., 2005). The characterization of the morphology, structure and properties in the latter type of layer is particularly complicated because of their strong three-dimensional local inhomogeneity and is normally carried out by electron microscopy, electrophysical and optical methods (Arsentyev et al., 2002, 2005; Nohavica, Gladkov, Jarchovský \& Zelinka, 2008; Nohavica, Gladkov, Jarchovský, Zelinka et al., 2008; Domashevskaya et al., 2008; Liu \& Duan, 2001; Tiginyanu et al., 2003; Föll et al., 2003).

X-ray analysis of porous buffer layers has been limited so far, mostly because of the difficulty of data interpretation. Nevertheless, X-ray diffraction methods have been used successfully, but in the main for analysis of porous silicon layers (Barla et al., 1984; Goudeau et al., 1989; Bensaid et al., 1991). It has been shown that crystalline quality and deformation of the porous layers depend on a lot of parameters, and in some cases the silicon skeleton persists as a result of the high quality of the bulk material and voids have various kinds and ordering. A numerical analysis of the specific features in the Bragg scattering from such structures was made by Lomov et al. (1995, 2009) and Buttard et al. (1998). It was shown that in a porous material with a high degree of crystal lattice coherence one can use the intensity of X-ray diffraction to obtain information on the thickness, deformation and statistical Debye-Waller factor, and on the degree of porosity in the layers. For the parameters of the pores and for crystallites that are displaced from coherent positions, only the form and angular spread of the diffuse scattering has been analyzed so far (Faivre \& Bellet, 1999). A quantitative analysis of pores, their geometrical form, fractality, ordering and space distribution was never implemented owing to the vast number of free parameters in the model.

\section{Diffuse X-ray scattering from porous crystalline layers}

The method of diffuse X-ray scattering has proved to be very effective for structural defect investigation (Huang, 1947; Dederichs, 1973; Krivoglaz, 1996). In the case of perfect crystals, the theory normally considers defects with nonoverlapping displacement fields, and the defects themselves are presented as point defects, spherical clusters or dislocation 
loops. In this case the total scattering is composed of two contributions: coherent Bragg scattering from the mean lattice and diffuse scattering from the defects. High-resolution X-ray diffraction (HRXD) allows one to study these components separately for different defect types (Iida \& Kohra, 1979; Kyutt et al., 1985; Lomov et al., 1985, 1999; Charniy et al., 1992). The diffuse scattering is of integral nature and provides an averaged scattering pattern, which makes the investigation of pores cumbersome owing to their variable size and form. A model taking into account the statistical variation of pore sizes in the kinematical approximation was presented by Punegov (2009). Meanwhile, the porous layers contain coherent crystallites (pore walls), so that the effects of multiple scattering must be also taken into account. In the case of porous layers, both the pores in the crystal lattice volume and the crystallites that have left the coherent positions belong to the structural defects. For a full description of scattering effects in such objects, one requires a statistical dynamical diffraction theory based on the approach described by Kato (1980).

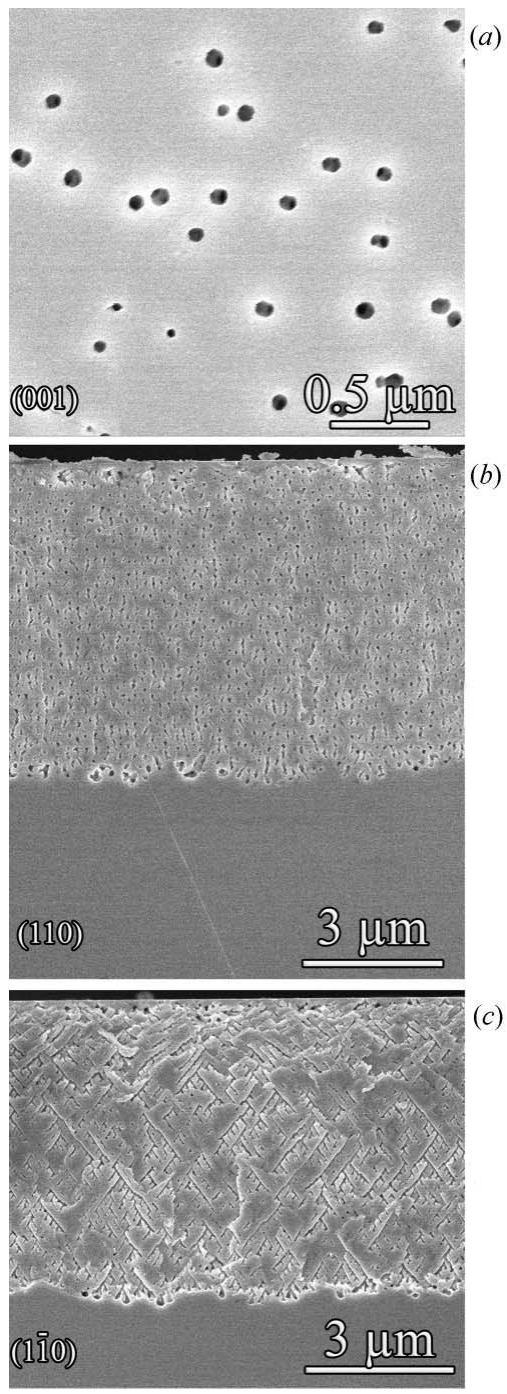

Figure 1

SEM images of the surface $(a)$ and the cleavage $(b),(c)$ of an $\operatorname{InP}(001)$ porous layer with pores oriented along the $\langle 111 B\rangle$ direction.
In this paper, we present an investigation of porous layers on an InP(001) substrate by a reciprocal space mapping method. The data evaluation was carried out by reciprocal space map (RSM) data fitting in the frame of the statistical dynamical diffraction model. The first systematic analysis of diffuse scattering from porous layers in InP was presented by Lomov et al. (2006, 2010) and Punegov et al. (2007). It was shown that high-resolution X-ray diffraction allows determination of the pore parameters, averaged over the sample volume. Lomov et al. (2010) used the X-ray diffraction method to investigate multilayer InP structures formed by pores of two different types. Punegov \& Lomov (2008) applied a model in the frame of the statistical dynamical diffraction theory to describe the diffuse scattering from cylindrical pores forming an angle $\alpha$ to the surface. Such porous structures, called crystallographically oriented, are typical for $A_{\mathrm{III}} B_{\mathrm{V}}$ materials and are formed under galvanostatic mode (Claussen et al., 2003; Delimitis et al., 2008; Tsuchiya et al., 2004; Ulin \& Konnikov, 2007). The basic crystallographic direction for the pore formation in indium phosphate by electrochemical etching is $\langle 111 B\rangle$. Depending on the applied voltage, lattice polarity and substrate doping type one can achieve pore formation in the directions $\langle 221\rangle,\langle 322\rangle$ and $\langle 111 A\rangle$, as well as along the surface normal. The inner surface of the pore is usually faceted along the $\{112\}$ and $\{111 A\}$ crystallographic planes, in accordance with the symmetry of the pore propagation direction (Claussen et al., 2003).

Diffraction and diffuse scattering from porous InP layers is clearly revealed as a two-dimensional intensity distribution in RSMs. However, in the RSMs published so far (Faivre \& Bellet, 1999; Punegov et al., 2007), only the basic features of scattering from the porous structures can be seen. This is primarily because of the low signal intensity from thin layers and insufficient data range. For investigation of scattering characteristics and recovery of detailed information on the pores and their layers, large data sets in a wide angular range with microradian resolution are required.

\section{Experimental}

Model porous layers were grown by electrochemical etching in galvanostatic mode on standard single-crystalline $\operatorname{InP}(001)$ substrates doped with $\mathrm{Sn}$ to the carried concentration $\sim 5 \times$ $10^{17} \mathrm{~cm}^{-3}$. The anodizing process was carried out in a fourelectrode configuration at $4 \mathrm{~V}$ electrode potential and $16 \mathrm{~mA}$ current, in the dark. $\mathrm{HCl}$ aqueous solution at $296 \mathrm{~K}$ was used as electrolyte.

Scanning electron microscopy (SEM) was used to control the thickness and structure of the porous layers. Samples were prepared by cleavage of the crystals parallel to the (110) and (1110) planes. A JSM 7401F (JEOL, Japan) electron microscope operated at $2-5 \mathrm{kV}$ was used for specimen analysis. Fig. 1 shows the surface $(a)$ and two cleavage planes $(b),(c)$ of a sample with a $\sim 6 \mu \mathrm{m}$ porous layer obtained by anodizing for a period of $100 \mathrm{~s}$. The surface image shows the etching pits as having an elongated faceted hexagonal shape. In the pitches, entrance openings of one or two pinholes predominantly 


\section{Table 1}

Characteristics of a porous buffer $\operatorname{InP}(001)$ layer with 'Christmas tree branch' architecture, recovered by mathematical fitting of experimental RSMs on the basis of the statistic dynamical theory for different azimuthal sample positions.

Reflection 004, Debye-Waller factor $f=0.6$ and porosity $P \leq 0.4$. 'No' indicates the absence of the correlation length for corresponding pores in the used model (HRXD) or the absence of visible correlation of corresponding pores in SEM images (although the pores are visible in the image).

\begin{tabular}{llll}
\hline & HRXD & \multicolumn{2}{l}{ SEM } \\
\hline Model of cylindrical pore & Long & Short & \multicolumn{2}{l}{ Stem } & Branch \\
Length of pore $l(\sigma)(\mathrm{nm})$ & $200(50)$ & $60(15)$ & $\sim 700 \sim 150$ \\
Cross section of pore $2 R_{\text {eff }}(\sigma)(\mathrm{nm})$ & $50(20)$ & $30(10)$ & $\sim 40 \sim 30$ \\
Lateral correlation length $L_{\mathrm{c}}[110](\sigma)(\mathrm{nm})$ & No & $140(35)$ & No $\sim 150$ \\
Lateral correlation length $L_{\mathrm{c}}[110](\sigma)(\mathrm{nm})$ & No & No & - \\
Inclination angle of pores $\alpha\left(^{\circ}\right) \dagger$ & $56(2)$ & & \multicolumn{2}{l}{$48-54$} \\
Layer thickness $L_{z}(\mu \mathrm{m})$ & $5.3(3)$ & $5.8(2)$ \\
Porosity $(\%) \ddagger$ & 0.4 & - \\
\hline
\end{tabular}

$\dagger$ Corresponding pores were not observed in SEM images. $\$$ Porosity was not calculated from SEM data.

oriented along preferred crystallographic directions can be observed. This is also confirmed by the images at cleavage planes. The porous layer looks sufficiently uniform over the whole thickness and anisotropic in pore orientation. On cleavage parallel to the (110) crystallographic plane (Fig. 1b), the pore exits have a triangular shape with the base oriented along the sample surface. On cleavage parallel to (110), which runs perpendicular to (110), the pores form branched patterns with a long central pore ('stem') and short side pores ('branches'). It is important to mention that the density of pores is

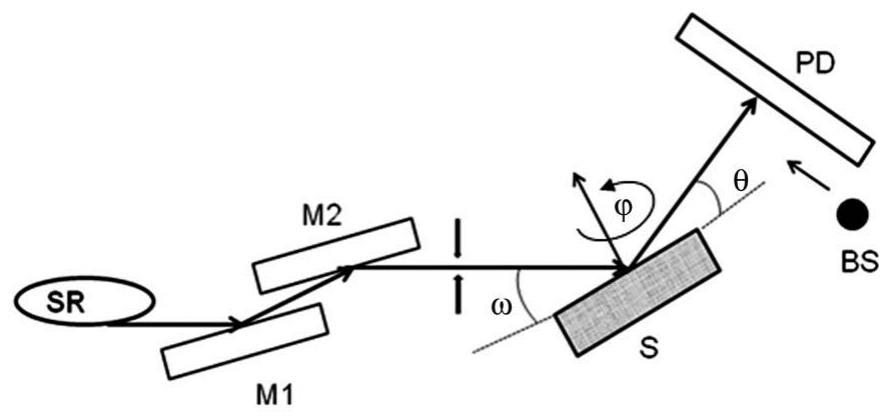

(a)

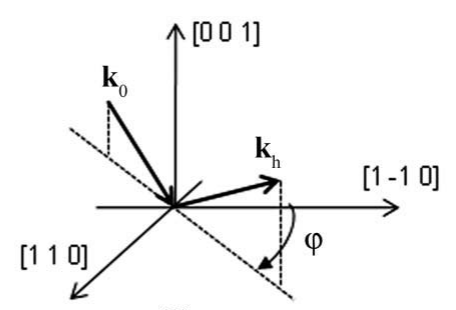

(b)

Figure 2

Experimental setup for high-resolution X-ray diffraction $(a)$ and positioning of scattering plane relative to the crystallographic axes of the sample at different azimuthal angles $\varphi(b) . \omega$ and $\theta$ are angular deviations from the exact Bragg positions for incident and reflected beams, respectively. SR: synchrotron radiation; M1,2: silicon singlecrystal monochromators; S: sample; PD: position sensitive detector; BS: beam stopper. higher than that of etch pitches. The mean values for the pore parameters are given in Table 1.

The X-ray diffraction measurements were carried out at the bending magnet beamline E2 at DESY. The layout of the experiment and the positions of the crystallographic planes relative to the scattering plane are shown in Figs. 2(a) and $2(b)$, respectively. The $10 \mathrm{keV}(\lambda=0.124 \mathrm{~nm})$ incident beam from the bending magnet was formed by a double-crystal $\mathrm{Si}(111)$ monochromator and a slit system. The exit slits of the collimator were set to $0.05 \mathrm{~mm}$ in the scattering plane and $2 \mathrm{~mm}$ the direction normal to it. The beam divergence in the scattering plane was $\Delta \theta \simeq 0.002^{\circ}$. The sample was adjusted to the 004 symmetric Bragg reflection. The scattered X-ray intensity $I(\omega, \theta)$ was registered by a MYTHEN linear position sensitive detector as a function of incidence angle $\omega$ and exit angle $\theta$, with scanning step $\Delta \omega=0.125^{\circ}$ and detector angular resolution $\Delta \theta=0.003^{\circ}$ channel. A beam stop was introduced in front of the detector at the incidence angles in the vicinity of the Bragg angle $\theta_{\mathrm{B}}$. The data acquisition time per point was set to $120 \mathrm{~s}$. The obtained data were used to build two-dimensional intensity maps $I(\mathbf{q}=\mathbf{Q}-\mathbf{h})$. Here, $\mathbf{Q}=\mathbf{k}_{\mathrm{h}}-\mathbf{k}_{0}$ is the scattering vector, $\mathbf{h}$ is the reciprocal lattice vector, and $\mathbf{k}_{0, \mathrm{~h}}$ are the wavevectors of the incident and scattered beams. The measurements were carried out at different azimuthal sample positions $\varphi=0,45$ and $90^{\circ}$, with $\varphi=0$ corresponding to $q_{x} \|$ [110]. The projections of the scattering vector $\mathbf{q}$ are given by

$$
\begin{aligned}
& q_{x}=k_{0}(2 \Delta \omega-\Delta \theta) \sin \theta_{\mathrm{B}}, \\
& q_{z}=k_{0} \Delta \theta \cos \vartheta_{\mathrm{B}} .
\end{aligned}
$$

X-ray mirror reflection curves were taken from the samples prior to diffraction measurements to control both surface corruption and the top surface layer density value. The reflectivity data show that the mean density of the $0.1 \mu \mathrm{m}$ top surface layers in porous samples is equal to that of the bulk InP. This is in good agreement with the SEM results (Fig. 1b), which show a dense $\sim 0.15 \mu \mathrm{m}$ layer in the surface area.

Experimental RSMs around the 004 reciprocal lattice node for the original InP substrate and for the InP sample with inclined pores are shown in Figs. 3 and 4. The reciprocal space maps allow the different components of the integral scattering to be separated, with some of them being well isolated and the others overlapping. In Fig. 3 one can distinguish the diffuse scattering structural imperfections in the bulk crystal, seen as smooth contours, and a number of sharper streaks (1-5) which arise as a result of the Bragg diffraction and the used experimental scheme: Bragg rod 1, pseudo-streak (Iida \& Kohra, 1979) 2, dispersive pseudo-streak 3 at $q_{z} / q_{x}=$ $2 \cot \theta_{\mathrm{Bm}}-\cot \theta_{\mathrm{Bs}}\left(\theta_{\mathrm{Bs}}\right.$ and $\theta_{\mathrm{Bm}}$ being the Bragg angles of the sample and monochromator, respectively), dynamical diffraction of diffuse scattering 4 , instrumental streak 5 . The angles between these streaks are connected to the experiment geometry. The parallelogram in the center of Figs. 3(a) and $3(b)$ comes from the beam stopper. The large variety of observed scattering components confirms the high quality of the original substrate material. It also proves that the multiple scattering effects must be taken into account both for the 
native InP bulk material and for the porous layer. Remarkably, the experimental data reveal not only the dynamical diffuse scattering of the incident and Bragg diffracted wave (Olekhnovich \& Olekhnovichi, 1981) but also interference effects on the strain fields caused by lattice defects (Aleksandrov \& Afanas'ev, 1984). At the same time, the interference effects are sensitive to anisotropy of strain fields near to the structural defects, as shown experimentally on porous GaAs structures (Lomov et al., 2012).

Experimental RSMs and $I\left(q_{x}\right)$ cross sections from the porous layer sample for different azimuthal angles are shown in Fig. 4 and in Figs. 7(a), 7(c) and 7(e) below. Two zones marked $\mathrm{N}$ and $\mathrm{E}$ can be outlined in the maps (Fig. 4). The

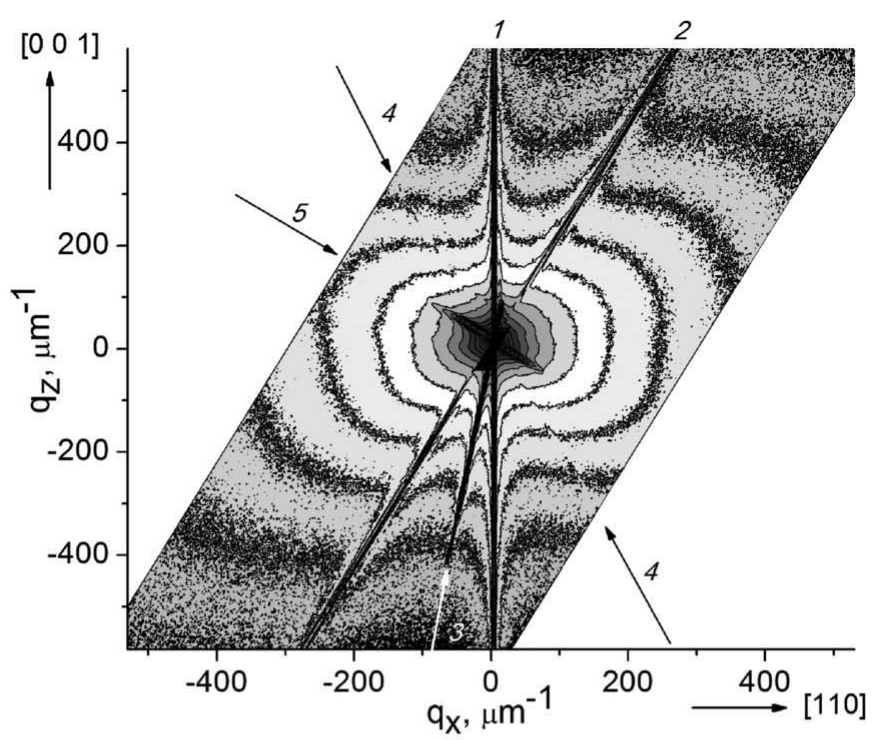

(a)

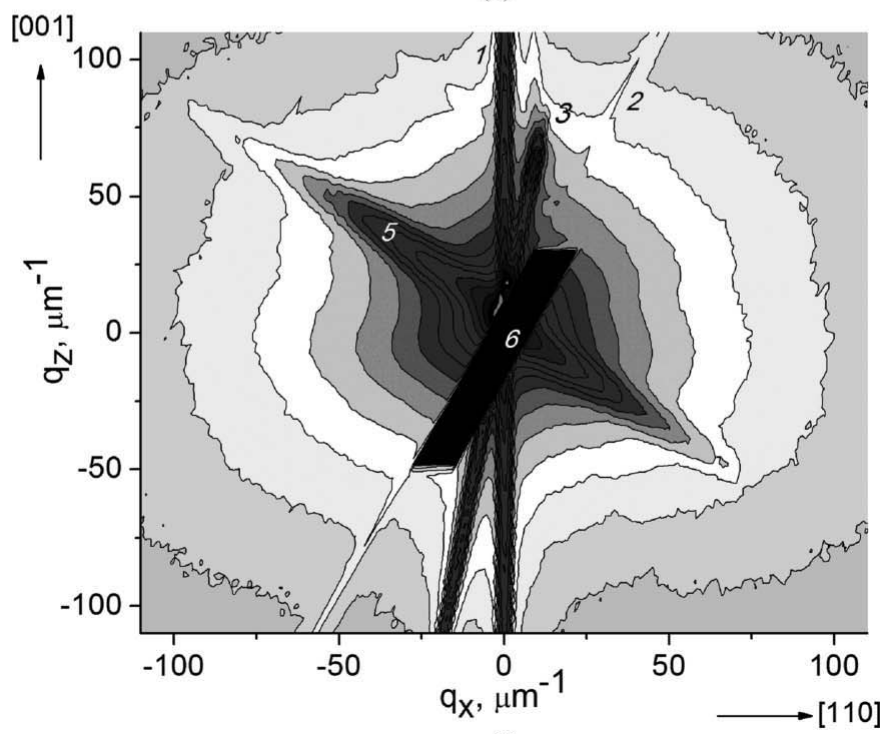

(b)

Figure 3

Original experimental RSM of an $\operatorname{InP}(001)$ substrate of total scattering from the 004 node on a small $(a)$ and large $(b)$ scale. The equi-intensity contours are shown in logarithmic scale, with step 0.786. Numbers 1-5 show features of the total scattering effects (for details see text). X-ray energy $E=10 \mathrm{keV}$, double-crystal $\mathrm{Si}(111)$ monochromator, position sensitive detector. The black band (6) in the RSM center comes from the beam stopper. diffuse scattering is dominated by the porous layer, which completely masks the component coming from point defects in the bulk. The diffuse scattering has a pronounced dependence on both the azimuthal angle $\varphi$ and scattering vector q. A quantitative analysis of the maps shows the presence of all of

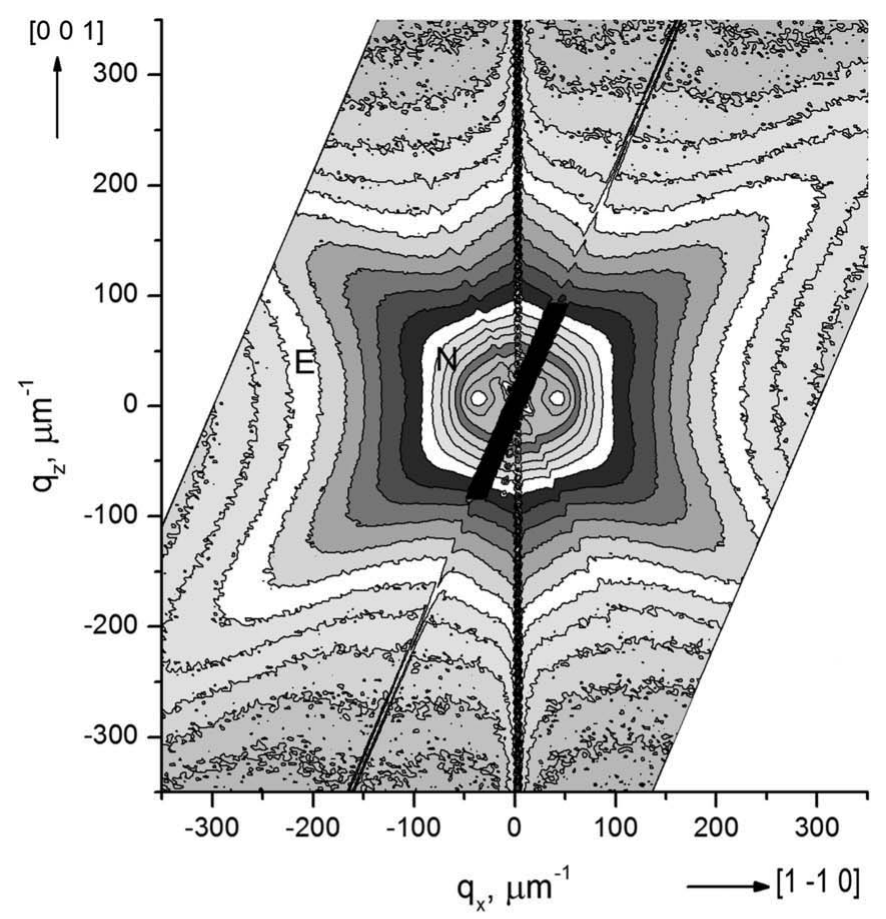

(a)

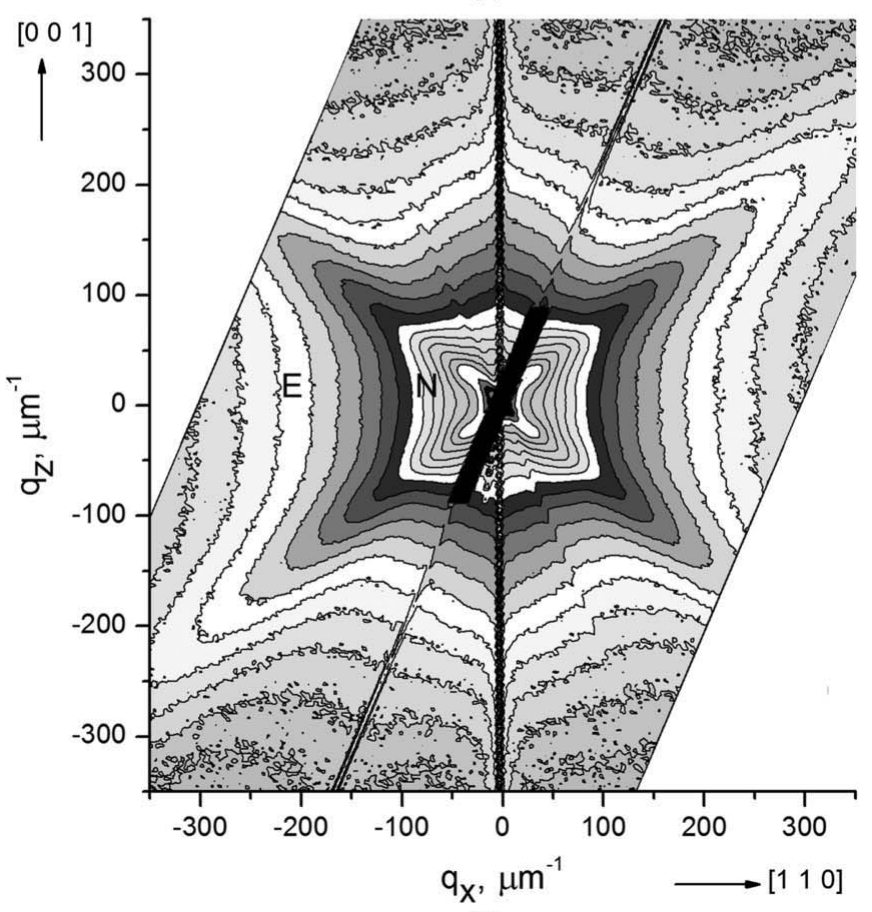

(b)

Figure 4

Original experimental RSMs of total scattering from an $\operatorname{InP}(001)$ porous layer near the 004 node with pores oriented along the $\langle 111 B\rangle$ direction at different azimuthal angles $\varphi=0^{\circ}(a)$ and $90^{\circ}(b)$. The equi-intensity contours are shown in logarithmic scale, with step 0.506. Different parts of the diffuse scattering are denoted by $\mathrm{E}$ and $\mathrm{N}$. For other details see text. 
the specific features discussed above, and these features are directly dependent on the form, dimensions and spatial ordering of the pores. With $\varphi$ variation, the form of the contour lines in the zone $\mathrm{N}$ gradually changes from ellipsoidal to cross formed. The two smooth maxima at the abscissa around $q_{x} \simeq \pm 38 \mu \mathrm{m}^{-1}$ correspond to correlation length $L_{\mathrm{c}}=$ $165 \mathrm{~nm}, L=2 \pi / q_{x}$. The maxima get less pronounced or completely disappear depending on the anisotropy of the pore's orientation and the contribution of the correlation term to the scattering intensity. A similar effect is well known in Bragg diffraction and mirror reflectivity on surfaces with correlated profiles. The correlation length value obtained from the data is defined along the line formed by the diffraction plane and the sample surface and in the case of ordered defects depends directly on the azimuthal angle $\varphi$. Fig. 5(a) and 5(c) show the $q_{x}$ cross sections with different correlation distances for $\varphi=0^{\circ}$ and $\varphi=45^{\circ}$. The maxima disappear completely for $\varphi=90^{\circ}$ (Fig. $5 e$ ), which matches the direction of pore ordering along [1]̄0].

The most pronounced features of the maps (Fig. 4) are the areas with elevated intensity, forming ridges at an angle $\beta \simeq$ $35^{\circ}$ to the $q_{x}$ direction. These ridges are the projections of the Fourier image of the scattering object, i.e. the pores, onto the scattering plane. The inclination angle of the ridges to the abscissae is directly related to the inclination of the pore walls to the sample surface, $\alpha=90^{\circ}-\beta$. Our experimental data show that the pores in the sample have formed predominantly along the [111] direction, as the value of $\alpha$ corresponds well to the known angle $54.7^{\circ}$ between the [111] and [001] directions. An azimuthal turn from $\varphi=90^{\circ}$ to $\varphi=0^{\circ}$ leads to the ridges vanishing from the $\mathrm{N}$ area. This indicates the presence of inclined pores and the breaking of fourfold lattice symmetry in the porous layer.

The azimuthal dependence changes take place at the boundary between the $\mathrm{N}$ and $\mathrm{E}$ zones at $\left|q_{x}\right| \simeq 100 \mu \mathrm{m}^{-1}$. From this, one can estimate the characteristic length of the pores, giving the main contribution to the scattering to be equal to $60 \mathrm{~nm}$. In zone $\mathrm{E}$, the form of the contour lines and ridges does not display any pronounced azimuthal angle dependence. This change indicates a very important alteration both in the ordering of long and short pore segments and in their space orientation.

Careful consideration of the RSMs permits us to draw a full model of the porous layer. First, let us consider the short segments that give the main contribution to the scattering and provide a strong azimuthal angle dependence. From Fig. 4(a), in zone $\mathrm{N}$ one can assume the presence of short segments formed along the [111] crystallographic direction and ordered

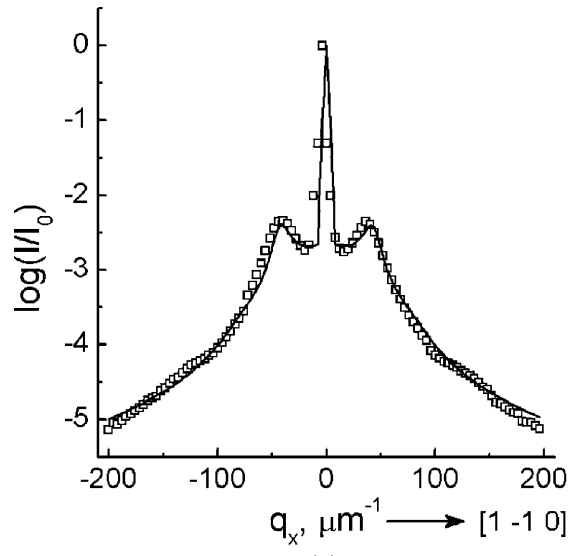

(a)

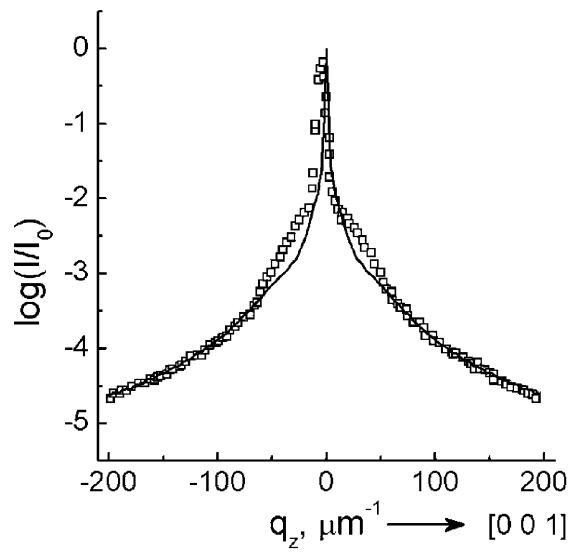

(b)

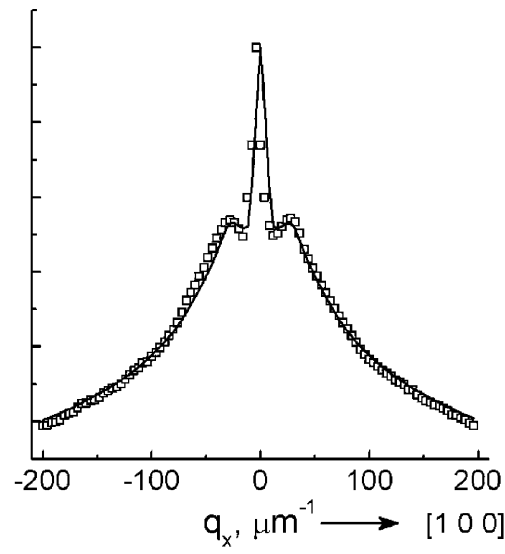

(c)

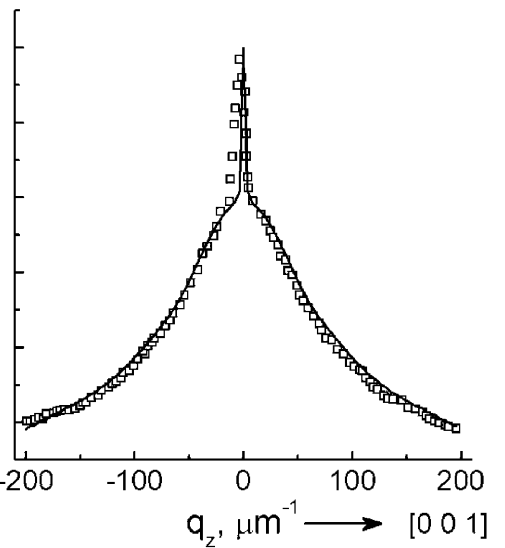

(d)

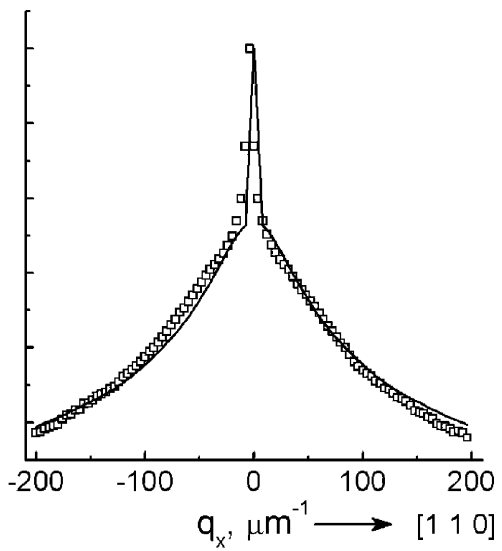

(e)

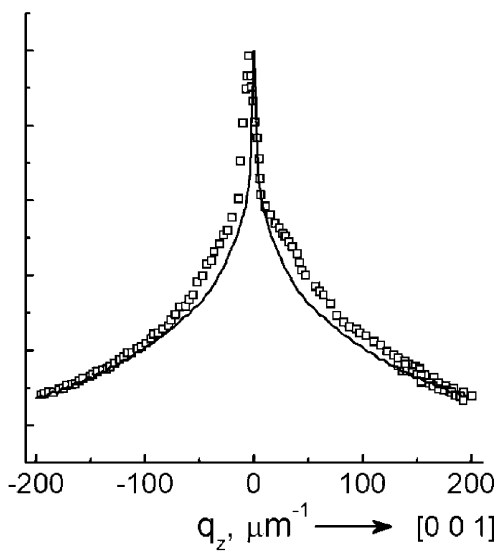

(f)

Figure 5

Examples of fitting scattering intensity cross sections near the $004 \mathrm{InP}$ reflection for a sample with a porous $6 \mu \mathrm{m}$-thick layer in different azimuthal positions: $\varphi=0^{\circ}(a),(b) ; \varphi=45^{\circ}(c),(d) ; \varphi=90^{\circ}(e),(f)$. 
along the surface in the [110] direction. This ordering is anisotropic and a rotation of the sample to $\varphi=90^{\circ}$ (Fig. $4 b$ ) leads to the disappearance of the correlation maximum. For this azimuthal position, the ridges both in the $\mathrm{N}$ zone and the $\mathrm{E}$ zone come from one and the same short segment type. These ridges disappear in Fig. 4(a), as can be seen in the $\mathrm{N}$ zone. Meanwhile, the ridges in the $\mathrm{E}$ zone indicate the presence of another type of short segment oriented along the [1111] direction. The number of pores with this second orientation is too low to provide any visible anisotropic features in the RSMs but must be still taken into account for correct numerical simulation. The same is true for any pores that are ordered along the surface normal and cannot produce clear and understood features in the RSMs. A thorough analysis of SEM images supports the presence two different directions for the short pore segments in the sample. Diffuse scattering from the long central pores (Fig. 1c) is focused near a reciprocal lattice node and contributes only a small fraction of the total scattering since their density is considerably less than that from the short pores.

The general analysis and estimates of pore dimension made above give a quantitative description of the porous layer structure. As already mentioned the SEM images show that the pores have a nonuniform distribution in space. We shall use this description to stress the possible differences in the parameters of pores defined by different methods. It will be necessary to take into account the form of the pores and the differences in size and direction for both groups.

\section{Dynamical diffraction on porous layers}

The statistical dynamical theory formalism developed by Kato (1980) allows simultaneous treatment of both coherent and diffuse scattering in crystals with broken translational crystal lattice order. In the frame of this approach, we shall first consider in detail the coherent Bragg scattering in a porous single-crystalline material and then identify the features of the diffuse scattering. The developed theory is applied to our numerical simulation and quantitative analysis of the structural characteristics of the investigated porous layer using experimental data.

\subsection{Coherent scattering}

Let us consider dynamical X-ray diffraction from a singlecrystalline sample with a homogeneous porous surface layer of thickness $L_{z}$, the coordinate axis $z$ along the inward normal to the surface and axis $x$ directed along the surface. The main structural parameters of the porous crystalline layer are the period of the diffracting planes $d$, the Debye-Waller factor $f$ and the layer porosity $P=1-v / v_{0}$, where $v_{0}$ and $v$ are the density of the bulk and porous material. In the presence of pores, the volume of the scattering medium decreases. Therefore, the factor $f$ in a general case can be presented as a product $f=f_{\mathrm{s}} f_{\mathrm{P}}$, where $f_{\mathrm{s}}$ corresponds to the defects of the skeleton crystalline structure of the porous layer, i.e. the defects of the crystal lattice and crystallites that have left their coherent positions, and $f_{\mathrm{P}}=(1-P)$ to the porosity due to the formation processing and interaction with air. The porous layer normally has a lattice constant different from that in the bulk, $\Delta d=d-d_{h k l}$, where $d_{h k l}$ is the interplanar distance in the bulk. Thus, in our analysis it is generally necessary to take into account the relative strain $\varepsilon=\Delta d / d_{h k l}$.

For simplicity, let us consider symmetric two-wave Bragg diffraction. The plane wave is incident on a porous layer under an angle $\theta=\theta_{\mathbf{B}}+\omega$, where $\theta_{\mathrm{B}}$ is the Bragg angle and $\omega$ is the deviation from the exact Bragg condition. X-ray scattering on crystals is commonly treated in reciprocal space, as the diffraction takes place on lattice planes that are normal to the reciprocal lattice vector $\mathbf{h}$, where $h=2 \pi / d_{h k l}$. The scattering intensity is defined by the susceptibility of the medium. For a perfect crystalline lattice, the Fourier component of the X-ray dielectric susceptibility is equal to $\chi_{h}=-r_{0} \lambda^{2} F_{h} /\left(\pi V_{\mathrm{c}}\right)$, where $F_{h}$ is the structure factor, $V_{\mathrm{c}}$ is the unit-cell volume, $r_{0}=e^{2} /$ $\left(m c^{2}\right)$ is the classical electron radius, and $e$ and $m$ are the charge and mass of an electron.

We should transform a system of Takagi (1969) equations for the case of Bragg diffraction geometry and our experimental scheme - the so-called triple-crystal diffractometry. This was discussed in detail by Punegov et al. (2010). For porous crystalline systems, the diffraction equations for the amplitude of the transmitted wave $E_{0}$ and diffracted wave $E_{h}$ take the form

$$
\left\{\begin{array}{l}
\frac{\partial E_{0}(z)}{\partial z}=i \bar{a}_{0} E_{0}(z)+i a_{-h} f E_{h}(z), \\
-\frac{\partial E_{h}(z)}{\partial z}=i\left(\bar{a}_{0}+\eta+\varepsilon h\right) E_{h}(z)+i a_{h} f E_{0}(z) .
\end{array}\right.
$$

Here we take into account the fact that the function $\mathbf{u}(\mathbf{r})$, describing the atomic displacements in the distorted crystal, can be represented as a sum of the mean and fluctuation components: $\mathbf{u}(\mathbf{r})=\langle\mathbf{u}(\mathbf{r})\rangle+\delta \mathbf{u}(\mathbf{r})$. The mean value of the phase function $\varphi(\mathbf{r})=\exp [(i \mathbf{h} \cdot \mathbf{u}(\mathbf{r})]$ can be then written as $\langle\varphi(\mathbf{r})\rangle=\bar{\varphi}(\mathbf{r}) f(\mathbf{r}), \quad$ where $\bar{\varphi}(\mathbf{r})=\exp [i \mathbf{h} \cdot\langle\mathbf{u}(\mathbf{r})\rangle]=\exp (i h \varepsilon z)$ describes the nonrandom long-range deformations in the bulk and $f(\mathbf{r})=\langle\exp [i \mathbf{h} \cdot \delta \mathbf{u}(\mathbf{r})]\rangle$ is the Debye-Waller factor. The angular distribution of the coherent component in equation (1) depends on the parameter $\eta=\left(2 \pi / \lambda \gamma_{h}\right) \sin 2 \theta_{\mathrm{B}} \omega$, which is connected to the projections of the vector $\mathbf{q}=\left(q_{x}, q_{y}, q_{z}\right)$ by $q_{z}=q_{x} \cot \theta_{\mathrm{B}}-\eta \cdot \gamma_{0, h}$ are the cosines of the angles between the normal to the crystal surface and the incident (0) and reflection $(h)$ direction, respectively. In symmetric Bragg geometry $\gamma_{0, h}=\left|\sin \theta_{\mathrm{B}}\right|$.

We shall further assume symmetric Bragg diffraction geometry and uniform pore distribution, as well as the absence of strain in the layer thickness $L_{z}$. For a freestanding porous film these conditions are $E_{0}(z=0)=1$ at the upper and $E_{h}\left(z=L_{z}\right)=0$ at the lower boundary. In that case, the solution for X-ray fields inside a porous film can be written as

$$
\begin{aligned}
& E_{0}(z)=\exp \left[i\left(\bar{a}_{0}+\xi_{2}\right) z\right]\left[\xi_{1} \exp \left(i \xi L_{z}\right)-\xi_{2} \exp (i \xi z)\right] / Q, \\
& E_{h}(z)=a_{h} f \exp \left[i\left(\bar{a}_{0}+\xi_{2}\right) z\right]\left[\exp \left(i \xi L_{z}\right)-\exp (i \xi z)\right] / Q,
\end{aligned}
$$




$$
\begin{gathered}
\xi_{1,2}=(-\bar{\eta} \pm \xi) / 2, \quad \xi=\left[(\bar{\eta})^{2}-4 a_{h} a_{-h} f^{2}\right]^{1 / 2} \\
Q=\xi_{1} \exp \left(i \xi L_{z}\right)-\xi_{2}, \\
a_{h,-h}=C \pi \chi_{h,-h} /\left(\lambda \gamma_{h, 0}\right)
\end{gathered}
$$

and $C$ is the polarization factor. The electromagnetic field amplitudes in the porous medium, taking into account the absorption and refraction effects, will depend on the angular variable $\bar{\eta}=2 \bar{a}_{0}+\eta$, where

$$
\bar{a}_{0}=(1-P) \pi \chi_{0} /\left(\lambda \gamma_{0}\right)+i a_{h} a_{-h}\left(1-f^{2}\right) \tau .
$$

Here, $\chi_{0}=-r_{0} \lambda^{2} F_{0} /\left(\pi V_{c}\right)$ is the Fourier component of the $\mathrm{X}$-ray dielectric susceptibility and $\tau=\tau(\eta)$ is a complex correlation length, describing the integral angular distribution of diffuse scattering in a double-crystal diffraction layout with wide detector aperture (Punegov, 1993). The first term of the coefficient $\bar{a}_{0}$ takes into account the reduction of charge density due to pores. The second term describes the additional refraction and absorption of X-rays due to local disruption of the exact translation periodicity of the crystalline lattice, caused by voids (statistically distributed pores) inside the medium, as well as structural distortions of the crystalline matter. One must note that, in Bragg geometry, the influence of that term is much lower than in Laue geometry.

For the amplitude reflection coefficient $r_{h}=E_{h}(z=0)$ one can directly derive from equation (4)

$$
r_{h}(\eta)=a_{h} f\left[\exp \left(i \xi L_{z}\right)-1\right] / Q .
$$

Contrary to the situation for diffraction on an ideal crystal, the dynamical coefficients $a_{h}$ and $a_{-h}$ in single-crystalline porous media are always multiplied by $f$. One should mention that the crystal porosity and the static Debye-Waller factor are linked, as the growth of pore concentration and/or their size lead to an increase of lattice imperfection. An analysis of this problem is given below.

If the porous layer is put on a substrate, at their interface the boundary condition is written as

$$
E_{h}\left(z=L_{z}\right)=E_{h}^{(\mathrm{sub})} .
$$

The solution of equation (9) allows us to calculate the diffraction curves in $\theta-2 \theta$ scanning mode with a wide detector aperture for the case of an unlimited plane wave incident on a porous layer. In that case, the coherent component of the diffracted intensity will form in reciprocal space a $\delta$-functionlike line along $q_{z}$. For the description of coherent scattering in triple-crystal diffractometry, a Fourier transformation of coherent fields in the lateral direction must be carried out (Punegov et al., 2010). Besides that, in a real experiment the incident beam is always limited in space. Therefore, the expression for the reflection coefficient amplitude will include a parameter dependent on the size of the beam footprint on the crystal surface $L_{x}$. In a real experiment, the incident beam is limited in space, has a final spectral range and a final angular divergence. Along with dispersion corrections, one must also take into account artifacts coming from the monochromator and the resolution of the linear detector. The full angular width of the intensity distribution in the reciprocal space is defined by the convolution of the incident radiation divergence with the reflections from the crystals in the optical layout; the intensity distribution can be described, for instance, by a Voigt or pseudo-Voigt profile $\Phi\left(q_{x}\right)$ (Ida et al., 2000).

To build an RSM, both coherent and diffuse components must be calculated. The intensity of coherent scattering in Bragg geometry is calculated according to

$$
I_{h}^{(\mathrm{c})}\left(q_{x}, q_{z}\right)=\left|r_{h}\left(q_{z}\right)\right|^{2} \Phi\left(q_{x}\right),
$$

where $r_{h}\left(q_{z}\right)$ is determined by formula (9) with $\eta=-q_{z}$.

\subsection{Diffuse scattering. Model of cylindrical pores}

For the analysis of diffuse scattering, the pores can be considered as a specific type of structural defect. Therefore, $\mathrm{X}$-ray diffraction on porous crystals is always accompanied by a noticeable diffuse scattering (Bensaid et al., 1991; Lomov et al., 1995; Faivre \& Bellet, 1999; Punegov et al., 2007). In the Bragg geometry, the dynamical interaction of scattered diffuse X-ray waves may be neglected (Punegov \& Kharchenko, 1998).

The expression for the intensity of diffuse scattering in the presence of spatially correlated pores has the form

$$
I_{h}^{(\mathrm{d})}(\mathbf{q})=\left|a_{h}\right|^{2}\left(1-f^{2}\right) T(\mathbf{q}) \int_{V_{0}} \mathrm{~d} \mathbf{r} \exp (-2 \mu z) I_{0}^{(\mathrm{c})}(\mathbf{r}, \mathbf{q})
$$

where $\mu$ is the linear absorption coefficient and $I_{0}^{(\mathrm{c})}(\mathbf{r}, \mathbf{q})$ the $\mathrm{X}$-ray beam intensity in the crystal volume with coordinate $\mathbf{r}$. We consider that diffuse scattering arises from a uniform porous crystalline layer with thickness $L_{z}$ and $V_{0}=S_{0} L_{z}$ is the full volume of the X-ray scattering, where $S_{0}=L_{x} L_{y}$ is the incident beam footprint.

$$
T(\mathbf{q})=\int_{-\infty}^{+\infty} \mathrm{d} \boldsymbol{\rho} G(\boldsymbol{\rho}) \exp (i \mathbf{q} \cdot \boldsymbol{\rho})
$$

is a parameter describing the angular distribution of the intensity, resulting from the form and space distribution of the pores, and $G(\boldsymbol{\rho})$ is the corresponding correlation function (Nesterets \& Punegov, 2000). One must note that the correlation function can be described by models involving the longor the short-range order. In the case of long-range order, the pores are fixed at equilibrium positions that have a strict translational order. For the short-range order, there exists a law defining the distribution of neighbors, without any periodicity rules. This was observed in experiments on porous silicon (Goudeau et al., 1989), porous germanium (Lomov et $a l ., 2003$ ) and porous indium phosphate (Punegov et al., 2007). If the distribution law for the nearest neighbors can be found, it will also allow definition of the full distribution function. The short-range order can be presented in the form of a radial distribution (Bushuev, 2007) or as a paracrystalline model (Hosemann, 1950; Punegov \& Lomov, 2008; Punegov, 2011).

The pores in a crystalline medium disturb in a random manner the strict translational order of the atom arrangement. 
This disorder can be described by random atomic displacements $\delta \mathbf{u}(\boldsymbol{\rho})$. Moreover, the elastic displacements $\delta \mathbf{u}(\boldsymbol{\rho})$ depend on the local coordinate $\mathbf{r}$. The elastic displacements caused by a single pore are included in the fluctuation phase factor $\exp [i \mathbf{h} \cdot \delta \mathbf{u}(\rho)]$. In the frame of Kato's statistical diffraction formalism a specific model of the pores is given by a correlation function, analogous to the form factor:

$$
g(\boldsymbol{\rho})=\frac{\langle\exp \{i \mathbf{h} \cdot[\delta \mathbf{u}(\boldsymbol{\rho})-\delta \mathbf{u}(0)]\}\rangle-f^{2}}{1-f^{2}} .
$$

As the angular distribution of the diffuse scattering intensity depends not only on the type of pores but also on their spatial distribution, the correlation function in equation (13) can be represented as a convolution of the pore distribution function $W(\boldsymbol{\rho})$ and the intrinsic correlation function $g(\boldsymbol{\rho})$ :

$$
G(\boldsymbol{\rho})=\int_{-\infty}^{\infty} \mathrm{d} \boldsymbol{\rho}^{\prime} W\left(\boldsymbol{\rho}^{\prime}\right) g\left(\boldsymbol{\rho}^{\prime}+\boldsymbol{\rho}\right)
$$

For randomly distributed pores, the correlation function $G(\boldsymbol{\rho})$ is directly transformed into the intrinsic correlation function $g(\boldsymbol{\rho})$.

Taking equation (13) into account and making use of the well known properties of Fourier transform convolution, the correlation volume can be represented by

$$
T(\mathbf{q})=\tau(\mathbf{q}) F(\mathbf{q}),
$$

where

$$
\tau(\mathbf{q})=\int_{-\infty}^{+\infty} \mathrm{d} \boldsymbol{\rho} g(\boldsymbol{\rho}) \exp (i \mathbf{q} \cdot \boldsymbol{\rho})
$$

is the Fourier transform of the self-correlation function for a single pore (pore correlation volume) and

$$
F(\mathbf{q})=\int_{-\infty}^{\infty} \mathrm{d} \boldsymbol{\rho} \exp (i \mathbf{q} \cdot \boldsymbol{\rho}) W(\boldsymbol{\rho})
$$

is the Fourier transform of the pore spatial distribution function (diffuse scattering interference structure factor). The correlation volume was introduced (Nesterets \& Punegov, 2000; Pavlov \& Punegov, 2000) in analogy to the common

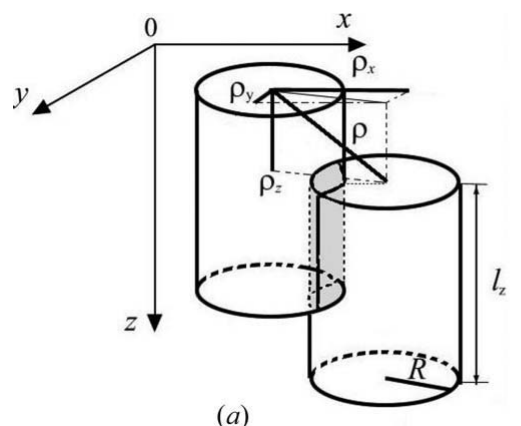

Figure 6

The model of cylinder pores with length $l_{z}$ and circular cross section with radius $R$. A geometric representation of the intersection of two cylinders (a) and the view from the cylinder top of the intersection (gray shading) of two circles $(b)$. correlation length. At a reciprocal lattice node $(q=0)$, the correlation volume becomes equal to the pore volume:

$$
\tau(0)=\int_{-\infty}^{+\infty} \mathrm{d} \rho g(\boldsymbol{\rho})=V_{\text {por }}
$$

Let us consider now a model medium with its crystalline order disturbed by randomly distributed cylindrical voids of radius $R$ and cylinder height $l_{z}$ (Fig. 6). The self-correlation function of such pores can be represented as

$$
g(\boldsymbol{\rho})=g_{0}\left(\rho_{x}, \rho_{y}, R\right) g_{z}\left(\rho_{z}, l_{z}\right) .
$$

Here the vertical correlation function along the surface normal has the form

$$
g_{z}\left(\rho_{z}, l_{z}\right)=\left\{\begin{array}{l}
1-\left|\rho_{z}\right| / l_{z}, \\
0,
\end{array} \mid \begin{array}{l}
\rho_{\mathrm{z}} \\
\rho_{\mathrm{z}} \mid>l_{z}
\end{array}\right.
$$

The two-dimensional lateral correlation function is given by

$$
\begin{array}{r}
g_{0}\left(\rho_{0}, R\right)=\frac{2}{\pi} \arcsin \left(1-\frac{\rho_{0}^{2}}{4 R^{2}}\right)^{1 / 2}-\frac{\rho_{0}}{\pi R}\left(1-\frac{\rho_{0}^{2}}{4 R^{2}}\right)^{1 / 2} \\
\text { for } \rho_{0} \leq 2 R
\end{array}
$$

and

$$
g_{0}\left(\rho_{0}, R\right)=0 \text { for } \rho_{0}>2 R .
$$

The statistical Debye-Waller factor

$$
f_{\mathrm{P}}=\exp \left(-c_{\mathrm{p}} \pi R^{2} l_{z}\right)
$$

depends on the pore size and concentration $c_{\mathrm{p}}$. Substituting (20) into (17) one obtains the expression for cylindrical pore correlation volume:

$$
\tau(\mathbf{q})=V_{\mathrm{p}} N\left(q, R, l_{z}\right)
$$

where

$N\left(\mathbf{q}, R, l_{z}\right)=\left[\frac{2 J_{1}\left(q_{0} R\right)}{q_{0} R}\right]^{2}\left[\operatorname{sinc}\left(\frac{q_{z} l_{z}}{2}\right)\right]^{2}, \quad \operatorname{sinc}(x)=\frac{\sin (x)}{x}$,

and $J_{1}$ is the first-order Bessel function. In our experiment the diffuse scattering is registered in reciprocal space. In this case, for numerical calculations one can use the correlation area

$$
\tau\left(q_{x}, q_{z}\right)=\int_{-\infty}^{\infty} \tau(\mathbf{q}) \mathrm{d} q_{y} .
$$

Theoretical calculations of the diffuse scattering angular distribution show that the intensity distribution along the projections $q_{x}$ and $q_{z}$ of the vector $\mathbf{q}$ in reciprocal space have an oscillatory structure. It is caused by the fast oscillatory behavior of the angular distribution function $N\left(\mathbf{q}, R, l_{z}\right)$. As a rule, experimental measurements of diffuse scattering carried out on porous single-crystal layers do not reveal any such oscillations. The absence of oscillation may be due to two factors: the presence of pores of different cross section and drawbacks of the experimental technique, mostly insufficient statistics. 
Here, following the formalism of Punegov (2009), let us take a statistical averaging over the pore size $L$, estimating the lognormal distribution

$$
\begin{array}{r}
p_{\mathrm{LN}}(L)=\frac{1}{(2 \pi)^{1 / 2} L \sigma_{\mathrm{LN}}} \exp \left\{-\frac{\left[\ln (L /\langle L\rangle)+\sigma_{\mathrm{LN}}^{2} / 2\right]^{2}}{2 \sigma_{\mathrm{LN}}^{2}}\right\} \\
(L \geq 0),
\end{array}
$$

where $\langle L\rangle=\int_{0}^{\infty} L p_{\mathrm{LN}}(L) \mathrm{d} L$ is the mean pore size in the vertical or lateral directions. The dispersion of the pore size $\sigma_{L}^{2}=\int_{0}^{\infty}(L-\langle L\rangle)^{2} p_{\mathrm{LN}}(L) \mathrm{d} L$ and the maximum position of the size distribution $L_{\max }$ convert to $\sigma_{L}^{2}=\left[\exp \left(\sigma_{\mathrm{LN}}^{2}\right)-1\right]\langle L\rangle^{2}$, $L_{\max }=\exp \left(-3 \sigma_{\mathrm{LN}}^{2} / 2\right)\langle L\rangle$. For small size variations, the distribution will become normal: $\sigma_{L}^{2} \cong \sigma_{\mathrm{LN}}^{2}\langle L\rangle^{2}, L_{\max } \cong\langle L\rangle$.

Ordered pores are characterized by short-range structural order, which can be described by a paracrystalline model (Hosemann, 1950; Eads \& Millane, 2001). For crystalline media without large-scale distortions and homogeneous in the lateral direction, the interference structure factor (18) will be written as

$$
\begin{aligned}
F\left(q_{x}, q_{y}\right) & =1 \\
+\frac{2}{N} & \operatorname{Re}\left(\frac{Z\left(q_{x}, q_{y}\right)\left\{\left[1-Z\left(q_{x}, q_{y}\right)\right] N-\left[1-Z\left(q_{x}, q_{y}\right)^{N}\right]\right\}}{\left[1-Z\left(q_{x}, q_{y}\right)\right]^{2}}\right),
\end{aligned}
$$

where

$$
Z\left(q_{x}, q_{y}\right)=\int_{-\infty}^{+\infty} \mathrm{d} \rho_{x} \int_{-\infty}^{+\infty} \mathrm{d} \rho_{y} \exp \left[i\left(q_{x} \rho_{x}+q_{y} \rho_{y}\right)\right] H_{1}\left(\rho_{x}, \rho_{y}\right),
$$

$N$ is the number of pores in a selected lateral direction and $H_{1}\left(\rho_{x}, \rho_{y}\right)$ is a function of the probabilistic location of the first neighbor pores in the lateral plane.

As the experimental data are recorded in the plane $q_{x} q_{z}$, the structure factor for the calculations should be taken as

$$
\bar{F}\left(q_{x}\right)=\int_{-\infty}^{\infty} F\left(q_{x}, q_{y}\right) \mathrm{d} q_{y} .
$$

The intensity of diffuse scattering near a reciprocal lattice node can be calculated with the help of

$$
I_{h}^{(\mathrm{d})}\left(q_{x}, q_{z}\right)=\left|a_{h}\right|^{2}\left(1-f^{2}\right) \tau\left(q_{x}, q_{z}\right) \bar{F}\left(q_{x}\right) \Phi\left(q_{x}, q_{z}\right) .
$$

The term

$$
\Phi\left(q_{x}, q_{z}\right)=\int_{-L_{x} / 2}^{L_{x} / 2} \mathrm{~d} x \int_{0}^{L_{z}} \mathrm{~d} z \exp (-2 \mu z) I_{0}^{(\mathrm{c})}\left(q_{x}, q_{z} ; x, z\right)
$$

takes into account the lateral size of the incident beam and the influence of primary extinction on the recorded signal through the angular and spatial variation of the transmitted beam $I_{0}^{(\mathrm{c})}\left(q_{x}, q_{z} ; x, z\right)$.

One should mention that the self-correlation volume $\tau\left(q_{x}, q_{z}\right)$ describes the diffuse scattering by single pores, whereas the interference structure factor $\bar{F}\left(q_{x}\right)$ represents the short-range order. For randomly distributed pores there is no interference diffuse scattering and $\bar{F}\left(q_{x}\right)=1$.

\section{Data evaluation and discussion}

The numerical modelling of the experimental RSMs from investigated samples is a complex task owing to the large number of observed scattering effects and to the great volume of experimental data. However, the accurate interpretation of experimental data allowed us to significantly simplify the calculations. Firstly, the dynamical scattering components are concentrated in narrow angular bands and have virtually no overlap with the diffuse scattering from pores. Secondly, the diffuse scattering from structural defects in the bulk is weak compared to that from pores and can be neglected. Finally, one can obviously discriminate the dynamical effects in the diffuse scattering from pores as the former have a very weak influence on the general form of the maps. This allows the reduction of the quantity of data that must be treated by map fitting.

The diffraction curve was calculated in a recurrent procedure, taking into account the diffraction on the layer and substrate with equation (11). The expression for X-ray diffuse scattering $I_{h}^{(\mathrm{d})}\left(q_{x}, q_{z}\right)$ is given in equation (32). The total intensity, including coherent and diffuse components, can be found by direct summation:

$$
I_{h}^{(\mathrm{t})}\left(q_{x}, q_{z}\right)=I_{h}^{(\mathrm{c})}\left(q_{x}, q_{z}\right)+I_{h}^{(\mathrm{d})}\left(q_{x}, q_{z}\right) .
$$

The approach described above was used by us for numerical simulation of X-ray scattering from porous crystals.

The fitting procedure was implemented by least-squares minimization of

$$
\rho=\frac{1}{M} \sum_{m=1}^{M}\left[\frac{I_{\exp }\left(q_{x, z}^{(\mathrm{m})}\right)-I_{\text {calc }}\left(q_{x, z}^{(\mathrm{m})}\right)}{I_{\text {exp }}\left(q_{x, z}^{(\mathrm{m})}\right)}\right]^{2} .
$$

Here $I_{\text {exp }}\left(q_{x, z}^{(\mathrm{m})}\right)$ and $I_{\text {calc }}\left(q_{x, z}^{(\mathrm{m})}\right)$ are the experimental and theoretical RSM intensity, $I_{\text {exp,calc }}\left(q_{x}^{(\mathrm{m})}, q_{z_{i}}\right)$ and $I_{\text {exp,calc }}\left(q_{z}^{(\mathrm{m})}, q_{x_{i}}\right)$ are cross sections of the RSMs over $q_{x}^{(\mathrm{m})}$ and $q_{z}^{(\mathrm{m})}$ for $\omega$ and $\omega-2 \theta$ scanning. $M$ is the dimension of the data array. To reduce the calculation time, we have rendered the intensity maps to $200 \times$ 200 arrays with equal angular steps. As a first step, the coherent component $I\left(q_{z}^{(\mathrm{m})}, x=0\right)$ with static Debye-Waller factor was calculated. Further on, the full (coherent and diffuse) scattering was fitted simultaneously for nine $I\left(q_{x}\right)$ and seven $I\left(q_{z}\right)$ sections of RSMs. The iterations were stopped when the residual $\rho$ became lower than $5-7 \%$ for all sections. The main variables were the porous layer thickness $L_{z}$, the statistical factor $f$, the pore concentration $N$, the radius $R_{\text {eff }}$, the branch length $l$, the correlation length $L_{\mathrm{c}}$ for all pore groups and the degree of spatial anisotropy. The scattering from long and short branches was assumed to be independent.

The calculation of RSMs was carried out for the $004 \mathrm{InP}$ reflection of $\sigma$-polarized X-ray radiation with wavelength $0.124 \mathrm{~nm}$. The Fourier component values of dielectric susceptibility were taken to be equal to $\chi_{0}^{\operatorname{InP}}=(-2.68+i 0.024) \times$ $10^{-5}$ and $\chi_{h}^{\mathrm{InP}}=(-1.46+i 0.02) \times 10^{-5}$ (Stepanov \& Forrest, 2008, http://sergey.gmca.aps.anl.gov/).

The fitted cross sections and maps for different azimuthal angles are shown in Figs. 5 and 7, respectively. The fits were 
made taking into account the presence of two groups of pores. Every group contained short and long pores. Among the short pores, $93 \%$ of them were directed along [111] and were correlated, while the $7 \%$ directed along [1111] were distributed randomly. Long pores of both groups were not correlated and were distributed along the [111] and [1111] directions in the same proportion as short pores. The total volume of the subsurface layer occupied by pores was divided between short and long pores in a ratio of nine to one.

The calculated cross sections along $q_{x}$ (Figs. $5 a, 5 c$ and $5 e$ ) show a very good agreement with experiment. The $q_{z}$ sections partially deviate from experiment. Most notably, this deviation is in the range $\pm 40 \mu \mathrm{m}^{-1}$ around the reciprocal space node for $\varphi=0$ and $90^{\circ}$. In contrast, at the angle $\varphi=45^{\circ}$ theory coincides well with experiment. We assume that our model of porous layers is not sufficiently correct, because the diffuse scattering from short segments of inclined pores partly ordered along the surface normal is not included, and the deformations in the crystalline skeleton of the porous layer are also ignored. In the
RSMs (Fig. 7), these inconsistencies are less visible owing to the dynamical range of the intensities.

The fitted RSMs at $\varphi=0$ and $90^{\circ}$ show a very good coincidence within the areas free of dynamical effects and experimental artifacts (Figs. $7 a, 7 b, 7 e$ and $7 f$ )

The model calculations show that the 'short branches' give the main contribution to the diffuse scattering. An explanation for that can be found in the electron microscopy results (Fig. 1b). The long branches are in reality formed by chains of segments with variable form and size, while the diffuse scattering is especially sensitive to any form variation. Therefore, the signal from correlated objects comes mainly from the more stable short pores.

Scattering from inclined pores is seen as ridges in the RSMs over the whole angular range. The inclination of the ridges to the coordinate axes for $\varphi=0$ and $90^{\circ}$ coincides, which confirms the pore orientation along the $\langle 111\rangle$ direction. However, at the azimuthal position $\varphi=45^{\circ}$ the inclination angle $\beta$ between the ridge and the $q_{x}$ axis increases to $48^{\circ}$, while a model for a pore $\left[\begin{array}{lll}0 & 0 & 1\end{array}\right]$

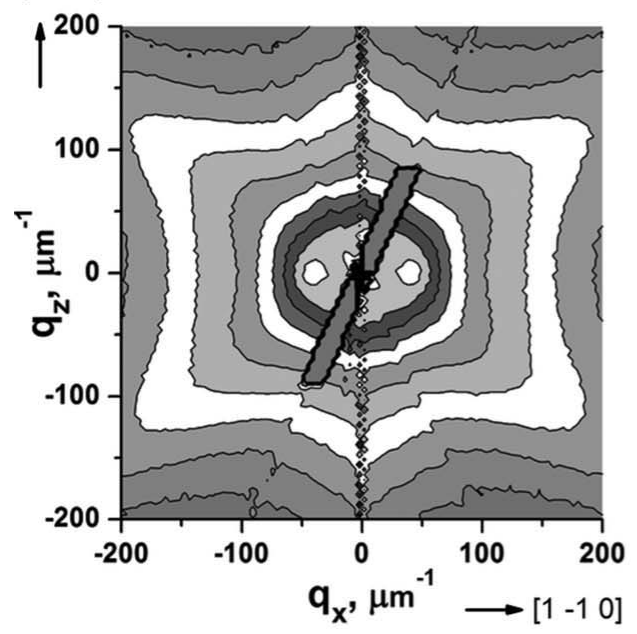

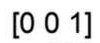

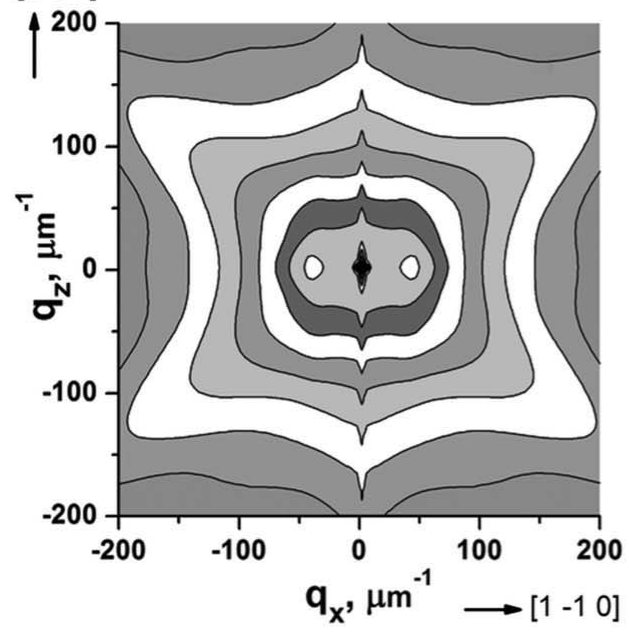

(b)

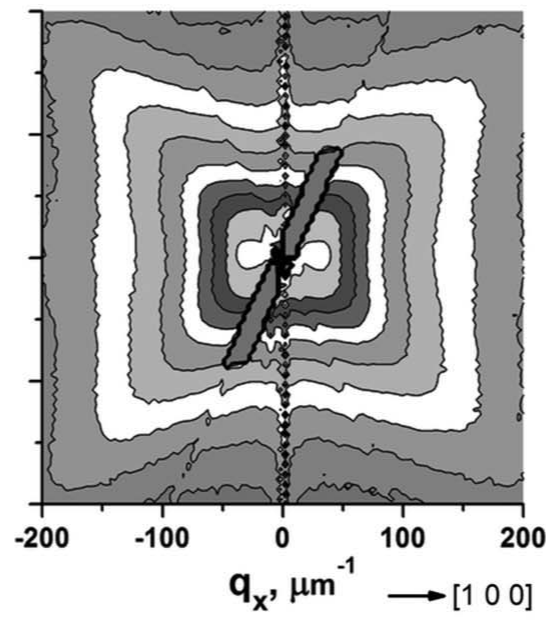

(c)

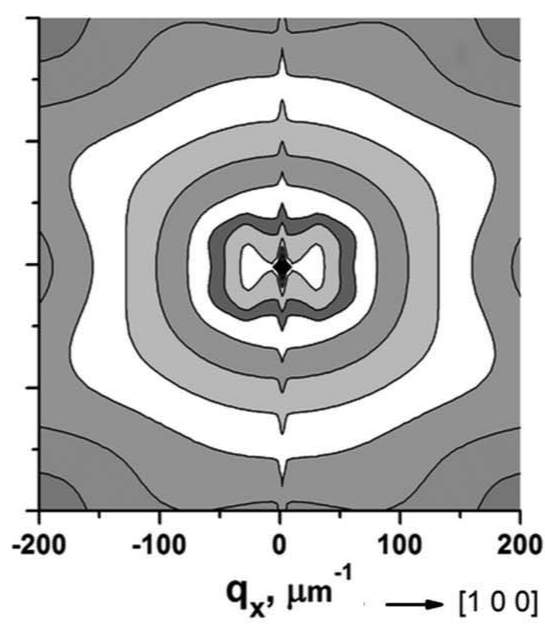

(d)

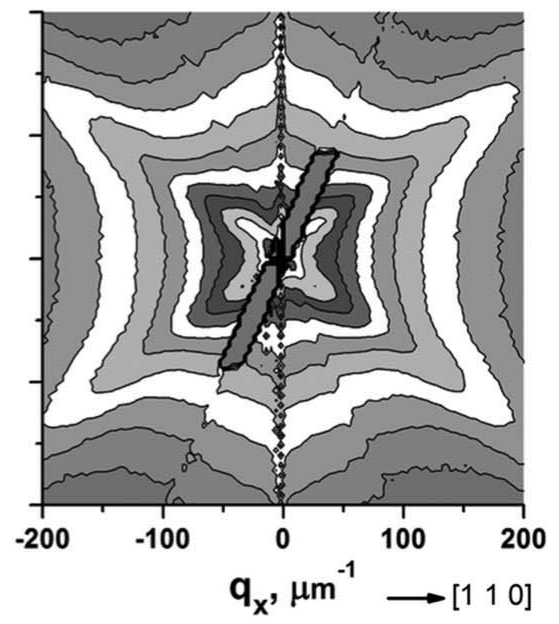

(e)

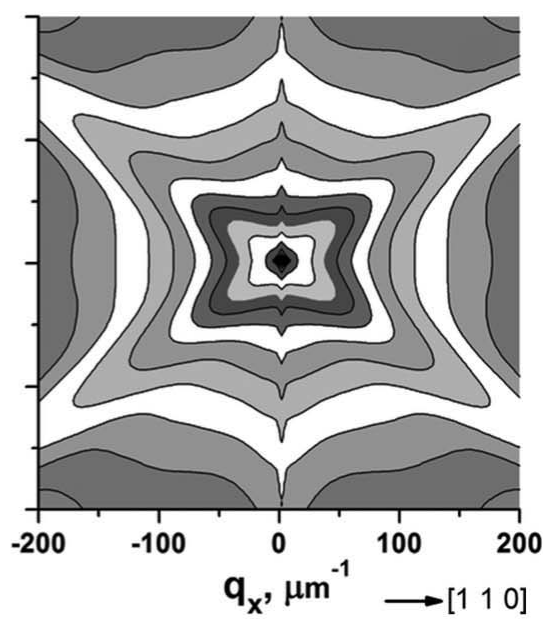

(f)

Figure 7

Experimental $(a),(c),(e)$ and theoretical $(b),(d),(f)$ reciprocal scattering maps for a sample with a porous $6 \mu$ m-thick layer in different azimuthal positions: $\varphi=0^{\circ}(a),(b) ; \varphi=45^{\circ}(c),(d) ; \varphi=90^{\circ}(e),(f) .004 \mathrm{InP}$ reflection, energy $E=10 \mathrm{keV}$. 
with negligible radius predicts the decrease of this angle to $\sim 26^{\circ}$. This feature clearly indicates that the form of the pores must necessarily be included in the model. The calculated map for $\varphi=45^{\circ}$, shown in Fig. $7(d)$, has two types of ridges with angles $\beta \simeq 25$ and $65^{\circ}$. The emergence of additional ridges has a simple geometrical explanation. In our model the projection of a pore onto the diffraction plane is approximated by a rectangle of length $l_{z}$ with base $2 R_{\text {eff }}$. The diffuse scattering, depending directly on the Fourier transform, would produce two types of ridges, which will vanish for $\varphi=0$ and $90^{\circ}$ correspondingly. In the case of inclined pores, the intersection of a pore with the scattering plane will form an ellipse or a truncated ellipse. In a real sample, the pores cylinders have a significantly fractionary form and contain all sorts of voids, which makes the RSM more complex.

Table 1 shows the main structural parameters defined from the X-ray scattering data and independently from the electron microscopy images. The best agreement is achieved for the thickness of the porous InP layer $L_{z}=5.3 \pm 0.3 \mu \mathrm{m}$, porosity $P=0.4$, Debye-Waller factor $f=0.60 \pm 0.05$ and negligible lattice deformation. The $L_{z}$ value obtained from X-ray data is about $0.5 \mu \mathrm{m}$ lower than that from SEM. This could be due to effects at the interface between the porous layer and the substrate. The obtained $R_{\text {eff }}$ values for the effective radii of long and short branches demonstrate a good agreement with the SEM results. A more detailed analysis for the $\varphi=0$ position also shows that the lateral correlation length $L_{[1 \overline{1} 0]}$ of the short branches is in very good agreement with the SEM images. The X-ray data provide, in addition, a quantitative value for the degree of pore anisotropy. This also proves that there is no ordering in the pore distribution at $\varphi=90^{\circ}$, i.e. along the [110] direction. The significant discrepancy in the pore length values obtained by different methods is caused by the difference in the underlying structure description. The SEM values are obtained by measuring the lengths of the pore slices visible in the images. The X-ray model, in contrast, takes into account the real segmented structure of single pore branches and considers the mean segment dimension as the basic parameter of the pore length.

\section{Conclusion}

To summarize, in this work we have used X-ray high-resolution reciprocal space mapping for the investigation of X-ray scattering from porous buffer InP layers with inclined pores. A theoretical model in the frame of the statistical dynamical theory for cylindrical pores was developed and applied for quantitative data evaluation. The comprehensive analysis of experimental data allowed us to investigate and describe theoretically the main dynamical and interference effects in diffuse X-ray scattering. The theoretical RSMs were fitted to experimental ones for different azimuthal positions of the sample. Mathematical treatment of the experimental data allowed us to extract the mean values for the main porous layer parameters such as porosity, form and pore orientation. It is shown that the RSM method can provide quantitative information in the case of nondeformed and buried porous layers that is not attainable with conventional diffraction and reflectometry methods. The results are in a good agreement with scanning electron microscopy data.

This study was supported in part by the Russian Foundation for Basic Research (projects No. 12-07-00745-a, No. 13-0200272-a), the Presidium of the Russian Academy of Sciences (project No. 12-P-1-1014), and the Ural Branch of the Russian Academy of Sciences (basic research program project No. 12U-1-1010).

\section{References}

Adachi, S. (1992). Editor. Physical Properties of III-V Semiconductor Compounds: InP, InAs, GaAs, GaP, InGaAs, and InGaAsP. New York: Wiley.

Aleksandrov, P. A. \& Afanas'ev, A. M. (1984). Kristallografia, 29, 1019-1021.

Arsent'ev, I. N., Baidakova, M. V., Bobyl', A. V., Vavilova, L. S., Konnikov, S. G., Ulin, V. P., Boltovets, N. S., Konakova, R. V., Milenin, V. V. \& Voitsikhovskii, D. I. (2002). Tech. Phys. Lett. 28, 735-739.

Arsentyev, I. N. et al. (2005). Semicond. Phys. Quantum Electron. Optoelectron. 8, 95-104.

Barla, K., Herino, R., Bomchil, G., Pfister, J. \& Freund, A. (1984). J. Cryst. Growth, 68, 727-733.

Bensaid, A., Patrat, G., Brunel, M., de Bergevin, F. \& Hérino, R. (1991). Solid State Commun. 79, 923-928.

Bushuev, V. A. (2007). J. Surf. Invest. X-ray Synchrotron Neutron Tech. 1, 522-527.

Buttard, D., Bellet, D., Dolino, G. \& Baumbach, T. (1998). J. Appl. Phys. 83, 5814-5822.

Charniy, L. A., Morozov, V. M., Bublik, V. T., Scherbachev, K. D., Stepantsova, I. V. \& Kaganer, V. M. (1992). J. Cryst. Growth, 116, 362-375.

Choi, H., Jeong, Y., Cho, J. \& Jeon, M. (2009). J. Cryst. Growth, 311, 1091-1095.

Claussen, J. C., Carstensen, J., Christophersen, M., Langa, S. \& Foll, H. (2003). Chaos, 13, 217-224.

Dederichs, P. H. (1973). J. Phys. P Metal Phys. 3, 471-496.

Delimitis, A., Komniou, Ph., Kehagias, Th., Pavlidou, E., Karakostas, Th., Gladkov, P. \& Nohavica, D. (2008). J. Porous Mater. 26, 75-81.

Domashevskaya, E. P., Kashkarov, V. M., Seredin, P. V., Terekhov, V. A., Turishchev, S. Y., Arsentyev, I. N. \& Ulin, V. P. (2008). Bull. Russ. Acad. Sci. Phys. 72, 439-442.

Eads, J. L. \& Millane, R. P. (2001). Acta Cryst. A57, 507-517.

Faivre, C. \& Bellet, D. (1999). J. Appl. Cryst. 32, 1134-1144.

Föll, H., Carstensen, J., Langa, S., Christophersen, M. \& Tiginyanu, M. (2003). Phys. Status Solidi A, 197, 61-70.

Goudeau, P., Naudon, A., Bomchil, G. \& Herino, R. (1989). J. Appl. Phys. 66, 625-628.

Hoke, W., Kennedy, T., Torabi, A., Whelan, C., Marsh, P., Leoni, R., Xu, C. \& Hsieh, K. (2003). J. Cryst. Growth, 251, 827-831.

Hosemann, R. (1950). Z. Phys. 128, 465-492.

Huang, K. (1947). Proc. R. Soc. London Ser. A, 190, 102-117.

Ida, T., Ando, M. \& Toraya, H. (2000). J. Appl. Cryst. 33, 1311-1316.

Iida, A. \& Kohra, K. (1979). Phys. Status Solidi A, 51, 533-542.

Katcki, J., Ratajczak, J., Adamczewska, J., Phillipp, F., Jin-Phillipp, N. Y., Regiński, K. \& Bugajski, M. (1999). Phys. Status Solidi A, 171, 275-282.

Kato, N. (1980). Acta Cryst. A36, 763-769.

Krivoglaz, M. A. (1996). X-ray and Neutron Diffraction in Nonideal Crystals. Berlin: Springer.

Kyutt, R. N., Sitnikova, A. A. \& Sorokin, L. M. (1985). Sov. Phys. Solid State, 27, 416-417. 
Landolt-Börnstein, R. (1982). New Series, Vol. 17, Subvol. A, Physics of Group IV Elements and III-V Compounds, edited by K.-H. Hellwege. Berlin: Springer-Verlag.

Liu, A. \& Duan, C. (2001). Appl. Phys. Lett. 78, 43-45.

Lomov, A. A., Bellet, D. \& Dolino, G. (1995). Phys. Status Solidi B, 190, 219-226.

Lomov, A. A., Bushuev, V. A., Imamov, R. M., Bocchi, C. \& Franzosi, P. (1999). Crystallogr. Rep. 44, 626-634.

Lomov, A. A., Bushuev, V. A., Karavanskii, V. A. \& Bayliss, S. (2003). Crystallogr. Rep. 48, 326-334.

Lomov, A. A., Bushuev, V. A., Kartsev, A. A., Karavanskii, V. A. \& Vasil'ev, A. L. (2009). Crystallogr. Rep. 54, 379-385.

Lomov, A. A., Chuev, M. A., Ulin, V. P., Vasiliev, A. L. \& Novikov, D. V. (2012). Book of Abstracts of the 11th Biennial Conference on High Resolution X-ray Diffraction and Imaging, 15-20 September, Saint-Peterburg, Russia, pp. 173-174.

Lomov, A. A., Prokhorov, D. Y., Imamov, R. M., Nohavica, D. \& Gladkov, P. (2006). Crystallogr. Rep. 51, 754-760.

Lomov, A. A., Punegov, V. I., Vasil'ev, A. L., Nohavica, D., Gladkov, P., Kartsev, A. A. \& Novikov, D. V. (2010). Crystallogr. Rep. 55, 182-190.

Lomov, A. A., Zaumseil, P. \& Winter, U. (1985). Acta Cryst. A41, 223-227.

Nesterets, Y. I. \& Punegov, V. I. (2000). Acta Cryst. A56, 540-548.

Nohavica, D., Gladkov, P., Jarchovský, Z. \& Zelinka, J. (2008). Acta Metall. Slovaca, 14, 240-246.

Nohavica, D., Gladkov, P., Jarchovský, Z., Zelinka, J., Komninou, P., Delimitis, A., Kehagias, T. \& Karakostas, T. (2008). Phys. Status Solidi A, 205, 2577-2580.
Olekhnovich, N. M. \& Olekhnovichi, A. I. (1981). Phys. Status Solidi A, 67, 427-433.

Pavlov, K. M. \& Punegov, V. I. (2000). Acta Cryst. A56, 227-234.

Punegov, V. I. (1993). Phys. Status Solidi A, 136, 9-19.

Punegov, V. I. (2009). Crystallogr. Rep. 54, 391-398.

Punegov, V. I. (2011). Tech. Phys. Lett. 37, 696-699.

Punegov, V. I. \& Kharchenko, A. V. (1998). Crystallogr. Rep. 43, $1020-1025$.

Punegov, V. I. \& Lomov, A. A. (2008). Tech. Phys. Lett. 34, 238240.

Punegov, V. I., Lomov, A. A. \& Shcherbachev, K. D. (2007). Phys. Status Solidi A, 204, 2620-2625.

Punegov, V. I., Nesterets, Y. I. \& Roshchupkin, D. V. (2010). J. Appl. Cryst. 43, 520-530.

Sitnikova, A. A., Bobyl, A. V., Konnikov, S. G. \& Ulin, V. P. (2005). Semiconductors, 39, 523-527.

Soldatenkov, F. U., Ulin, V. P., Yakovenko, A. A., Fedorova, O. M., Konnikov, S. G. \& Korolkov, V. I. (1999). Tech. Phys. Lett. 25, 852854.

Stepanov, S. \& Forrest, R. (2008). J. Appl. Cryst. 41, 958-962.

Takagi, S. (1969). J. Phys. Soc. Jpn, 26, 1239-1253.

Tiginyanu, I. M., Kravetsky, I. V., Langa, S., Marowsky, G., Monecke, J. \& Föll, H. (2003). Phys. Status Solidi A, 197, 549-555.

Tsuchiya, H., Hueppe, M., Djenizian, T., Schmuki, P. \& Fujimoto, S. (2004). Sci. Technol. Adv. Mater. 5, 119-123.

Ulin, V. P. \& Konnikov, S. G. (2007). Semiconductors, 41, 832-844.

Zeng, Y., Cao, X., Cui, L., Kong, M., Pan, L., Wang, B. \& Zhu, Z. (2001). J. Cryst. Growth, 227-228, 210-213. 